Le développement de la représentation de l'espace à Tahiti : variabilité du côté mer et du côté montagne

Bertrand Troadec

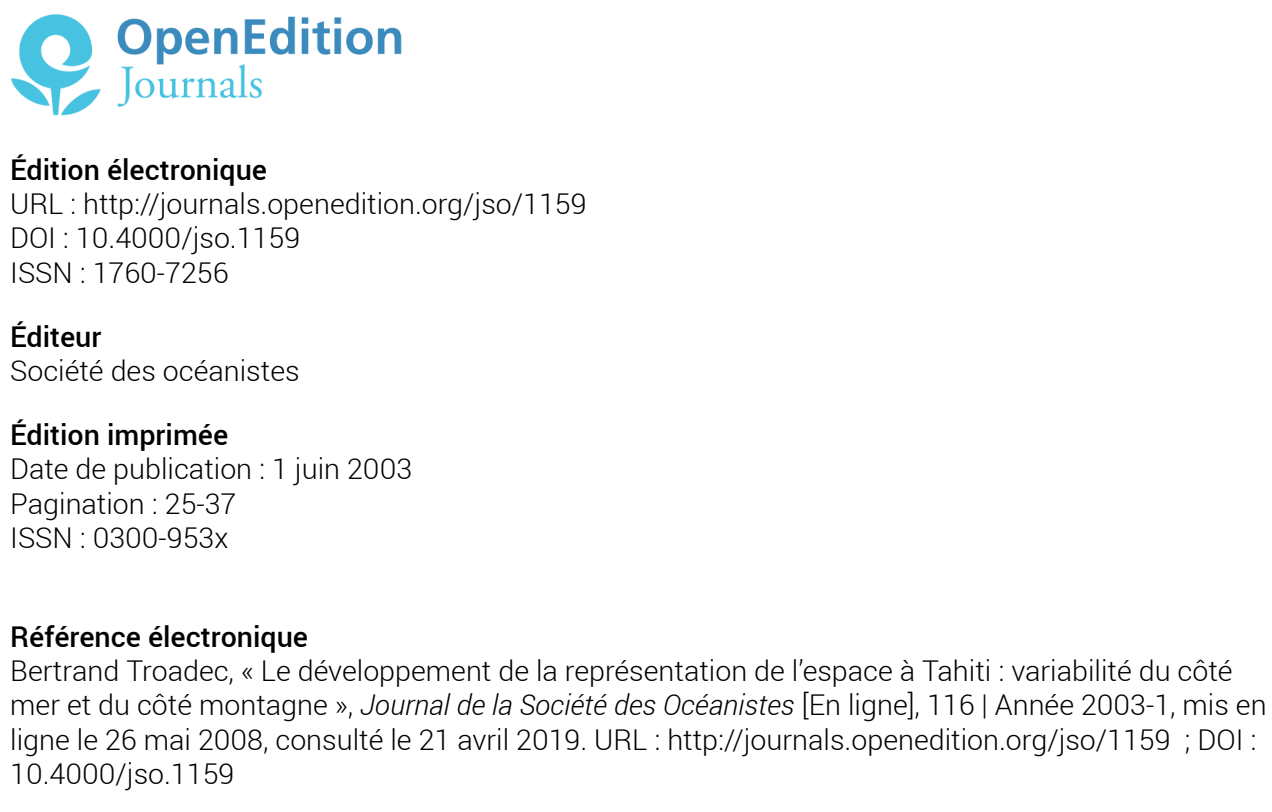

() Tous droits réservés 


\title{
Le développement de la représentation de l'espace à Tahiti : variabilité du côté mer et du côté montagne
}

\author{
par
}

\author{
Bertrand TROADEC ${ }^{*}$
}

\section{RÉSUMÉ}

Des travaux récents, d'inspiration anthropologique et linguistique, ont montré que le développement des systèmes de représentation de l'espace serait soumis à un "relativisme linguistique modéré ». Il s'ensuivrait la possibilité d'une "inversion des stades classiques du développement » dans certaines cultures. Cette interprétation pose le délicat problème de l'inférence d'une compétence cognitive supposée être "dans la tête» des sujets à partir des performances observées (action ou langage, ou les deux). En effet, évoquer un relativisme linguistique "modéré" sur la base d’une inversion "radicale » des étapes connues de l'ontogenèse n'apparaît pas épistémologiquement satisfaisant.

L'hypothèse que l'on se propose de mettre à l'épreuve des faits est qu'il ne s'agit pas d'une inversion des stades classiques de développement mais qu'un héritage historique et culturel particulier produit, de nos jours, des modalités de représentation de l'espace "inédites 》 (au moins, pour un chercheur occidental), dont les étapes du développement restent à décrire. Cette hypothèse est discutée par la présentation et l'analyse de données recueillies auprès d'un échantillon d'enfants tahitiens à qui l'on a fait passer deux épreuves relatives à l'orientation spatiale d'objets (Polynésie, Océanie).

Mots-CLÉs : relativisme linguistique, développement cognitif, espace relatif, espace absolu, Polynésie française, Océanie.

\begin{abstract}
Recent research, in anthropology and linguistics, showed that the development of children's spatial representation is bound by a "moderate linguistic relativism ». This leads to the possibility of a reversal of traditional stages of cognitive development in some cultures. The main question is how to infer cognitive competence from observed performance (i.e. action andlor language), competence which is unobservable and supposed to be "in the head" of subjects. Indeed, the evocation of moderate linguistic relativism as the basis of a reversal of known ontogenetic stages does not appear satisfactory from an epistemological point of view.

We propose that traditional (i.e. Western) ontogenetic stages are not being reversed but rather that a historical and cultural heritage produces, nowadays, new kinds of spatial representation. The stages of their development remain to be described by developmental psychologists. This assumption is discussed in the light of recent data obtained from two spatial orientation tasks realized with two samples of Polynesian children (Tahiti and Moorea, French Polynesia, Oceania).
\end{abstract}

KeYwords : Linguistic relativism, cognitive development, relative space, absolute space, French Polynesia, Oceania.

*. Université de Toulouse-Le Mirail (UTM), UFR de Psychologie, Département de Psychologie du Développement ; 5, allée Antonio Machado 31058 - Toulouse _Cedex 9 ; Tél./fax: 05.61.40.76.93 ; email : troadec@univ-tlse2.fr

Ce texte est une version écrite d'une communication orale intitulée «De la variabilité interindividuelle à la variabilité interculturelle : l'exemple du développement de la représentation de l'espace chez l'enfant tahitien ", présentée au VIII ${ }^{\mathrm{e}}$ Congrès international de l'Association pour la Recherche InterCulturelle (ARIC), à Genève, le 25 septembre 2001. Les trois recherches présentées composent un projet intitulé « Développement cognitif, représentations de l'espace, contextes culturels », subventionné dans le cadre du Programme «Cognitique » du ministère français de l'Éducation nationale, de la Recherche et de la Technologie. 
Dans l'ouvrage qui raconte sa propre découverte de l'île de Tahiti, en Océanie, Mazelier (vers 1965) écrit la chose suivante: "la maison de Mama Terii, son fare, est sise sur un étroit terrain au bout d'un chemin qui part 'côté montagne' de la route. 'Côté montagne', 'côté mer', sont des notions plus précises que 'à droite' ou 'à gauche' dans une contrée où l'unique route est circulaire » (p. 25). Notre objectif est de montrer l'importance actuelle, en Polynésie, de ce mode d'orientation dans l'espace, qualifié de « géocentré », par rapport à l'orientation dite « égocentrée » (droite/gauche) plutôt caractéristique des cultures occidentales. Toutefois, on propose surtout d'en analyser le développement chez l'enfant et d'examiner les relations entre l'action d'orienter des objets dans l'espace et le langage utilisé pour l'exprimer.

Ainsi, lors d'un séjour récent en Polynésie, on a pu entendre dans la cour d'une école primaire, à Tahiti, une hygiéniste dentaire apprenant à des enfants de Cours Préparatoire (CP) à se brosser les dents, dire (en français) : « Brossez... Maintenant, crachez... Puis, à gauche !... Côté route... ». Ceci suggère bien l'utilisation actuelle, en Polynésie et dans le cadre d'un contexte d'apprentissage formel (l'école), de référents géocentrés (la route) pour signifier une direction relative au corps (la gauche). Se brosser les dents qui sont du côté de la route peut alors surprendre considérablement un observateur de culture française, non averti. D'un point de vue anthropologique,
Ascher (1998: 183-184) estime effectivement que, « selon ce qu'elle considère comme important, chaque culture pose ses propres conventions spatiales et temporelles. Pour les gens de chaque culture, la structuration physique et conceptuelle qu'ils se donnent de l'espace-temps constitue une part si intégrante de leur monde et de leur vision du monde qu'elle semble tout à la fois évidente et naturelle ».

\section{RELATIVISME LINGUISTIQUE ET REPRÉSENTATION DE L'ESPACE}

Pour ce qui concerne une typologie des descripteurs linguistiques relatifs à l'orientation dans l'espace, on relève habituellement (voir le tableau 1) :

- des descripteurs égocentrés ou relatifs au locuteur (niveau 1) qui font, par exemple, référence aux côtés droit et gauche du corps propre et aussi exocentrés lorsqu'il s'agit du corps d'autrui et d'objets ou intrinsèques à l'auditeur et aux objets (niveau 2) ;

- des descripteurs géocentrés ou absolus (niveau 3) qui renvoient aux points cardinaux ou bien à des repères géographiques plus ou moins proches du locuteur, mais indépendants des sujets et des objets.

\begin{tabular}{|l|l|l|l|}
\hline Théories & Niveau 1 & Niveau 2 & Niveau 3 \\
\hline Linguistique (1) & $\begin{array}{l}\text { Relatif } \\
\text { (relative) }\end{array}$ & $\begin{array}{l}\text { Intrinsèque } \\
\text { (intrinsic) }\end{array}$ & $\begin{array}{l}\text { Absolu } \\
\text { (absolute) }\end{array}$ \\
\hline Linguistique (2) & $\begin{array}{l}\text { Déictique } \\
(\text { deictic })\end{array}$ & $\begin{array}{l}\text { Intrinsèque } \\
\text { (intrinsic) }\end{array}$ & $\begin{array}{l}\text { Extrinsèque } \\
\text { (extrinsic) }\end{array}$ \\
\hline Psychologie & $\begin{array}{l}\text { Égocentré } \\
(\text { egocentric })\end{array}$ & $\begin{array}{l}\text { Exocentré } \\
\text { (exocentric) }\end{array}$ & $\begin{array}{l}\text { Géocentré } \\
\text { (geocentric) }\end{array}$ \\
\hline $\begin{array}{l}\text { Épistémologie } \\
\text { génétique }\end{array}$ & $\begin{array}{l}\text { Topologique } \\
\text { (topological) }\end{array}$ & $\begin{array}{l}\text { Projectif } \\
\text { (projective) }\end{array}$ & $\begin{array}{l}\text { Euclidien } \\
\text { (Euclidian) }\end{array}$ \\
\hline
\end{tabular}

TABLEAU 1. - Lexique relatif à l'espace (d'après Dasen, Mishra et Niraula, 2000)

On précise qu'il est possible de différencier aussi plusieurs types de descripteurs linguistiques relatifs à l'espace géocentré. Par exemple,
Pederson (1993, cité par Dasen et al., 2000) en propose les cinq suivants : 
- A. Nord-Sud-Est-Ouest (NSEw); vers la mousson (monsoonwards) ; vers le couchant-vers le levant (towards sunset-sunrise);

- B. Amont-aval (uphill-downhill);

- C. Côté montagne-côté mer (inlandseaward) ;

- D. Points de repères conventionnels (conventional landmarks) utilisés pour désigner une direction lointaine (par exemple, "vers la maison du chef »);

- E. Points de repères locaux (local landmarks) utilisés pour désigner une direction dans un espace proche (par exemple, "vers la fenêtre »). Ce type de descripteurs est le seul parmi les cinq qui n'apparaît pas totalement indépendant de la position du locuteur.

Selon Ozanne-Rivierre (1997), bien que les termes anthropocentriques (ou égocentrés) existent dans toutes les langues, dans la plupart des langues austronésiennes, « les repères d'orientation fondamentaux ne sont pas les axes remarquables du corps humain mais sont extérieurs à l'individu, inscrits dans le monde naturel environnant $»($ p. 81). Ainsi, le système d'orientation proto-austronésien (malais, malgache, balinais, mélanésien, polynésien, etc.), tel qu'il peut être reconstitué, apparaît être un système fondé sur l'opposition fondamentale «terre/mer». Il apparaît directement lié au milieu écologique insulaire dans lequel le système linguistique s'est formé. En Océanie, de nos jours, il semble que « les repères [spatiaux] restent essentiellement géocentriques plutôt qu'anthropocentriques, qu'on soit dans l'espace large ou dans l'espace restreint » (p. 89). Ceci invaliderait le caractère « naturel » et « universel » du système d'orientation égocentré, caractéristique des langues indoeuropéennes, et pose la question de l'acquisition de ces systèmes par l'enfant. Pour résumer, si les descripteurs égocentrés et exocentrés apparaissent utilisés couramment dans les langues indoeuropéennes, il existe des langues dans lesquelles ce sont des descripteurs géocentrés qui sont utilisés préférentiellement, voire même de façon exclusive. Dans d'autres cas, les trois types de descripteurs peuvent être utilisés selon les contextes (rapports directs au corps ou bien au-delà) avec par exemple, une préférence pour les descripteurs géocentrés (Wassmann, 1994).

Pour ce qui est plus spécifiquement du tahitien (reo tahiti), Peltzer (1998) évoque différentes expressions spatiales relatives apparemment à un espace égocentré voire exocentré (p. 11). ('atau) ;
- Axe vertical-sur, dessus (ni'a) versus bas, en bas (raro) ;

- Axe frontal-devant (mua) versus derrière (muri).

Si la gauche et la droite semblent rendre compte de directions uniquement égocentrées et exocentrées, il n'en est pas de même pour les deux autres. En effet, celles-ci peuvent référer à la fois à un espace égocentré et géocentré (p. 14 et p. 19).

- Direction du levant (ni'a) versus direction du couchant (raro) ;

- Ce qui vient en premier (тиа) versus ce qui vient en dernier (muri).

Le système linguistique tahitien relatif à l'espace apparaît alors, selon Peltzer (1998), «lococentrique » plutôt qu' « égocentrique» dans la mesure où les sujets, les objets et l'espace dans lequel ils se tiennent sont indissociables. «C'est plus un système lococentrique qu'égocentrique, où la personne a un lien étroit avec l'espace, et où elle peut même être le lieu de l'action, de l'événement ou l'action elle-même (prédicat) » (p. 25).

Des travaux récents de linguistique, relatifs à la représentation de l'espace, ont posé la question de la nature des rapports entre les variations reconnues des structures linguistiques (c'est-àdire les langues) et la cognition non linguistique (c'est-à-dire l'action ou la pensée) (Bowerman et Levinson, 2000 ; Brown et Levinson, 2000 ; Danziger, 1997, 2001 ; Levinson, 1996). Il semble que l'on puisse affirmer qu'une différence linguistique peut être associée, dans une certaine mesure, à une différence non linguistique. Parmi les questions qui restent en suspens, il y a celles relatives à l'incidence des facteurs écologiques et culturels sur les représentations spatiales, et surtout à leur développement. Traditionnellement, ces questions relèvent de la linguistique et de l'anthropologie. Notre recherche tente d'y répondre d'un point de vue psychologique. Celui-ci implique, outre une compréhension de la phylogenèse, une analyse de l'ontogenèse et de la psychogenèse, c'est-à-dire du développement en micro-contextes (Troadec, 1998, 2001a, 2001b, à paraître).

\section{DÉVELOPPEMENT COGNITIF ET ORIENTATION SPATIALE}

Dans une recherche originale, Wassmann et Dasen (1998) ont montré que le système d'orien- 
tation spatiale de la culture balinaise (Indonésie, Asie du Sud-Est), lorsqu'il est utilisé pour résoudre des tâches de résolution de problèmes, est caractérisé par l'utilisation très précoce, dès 4-5 ans, de références géocentrées (niveau $3 \mathrm{du}$ tableau 1) plutôt qu'égocentrées (niveau $1 \mathrm{du}$ tableau 1). Des résultats similaires ont été obtenus par Cottereau-Reiss (1999, 2002) auprès d'enfants kanak en Nouvelle-Calédonie. Ces résultats soulèveraient le vieux problème du relativisme linguistique, c'est-à-dire l'hypothèse "Sapir-Whorf et Korzybski ». Celle-ci définit « le langage comme classificateur et organisateur de l'expérience sensible » (Baudelot, 1991 : 651). De ce point de vue, la question est de savoir jusqu'à quel point le fonctionnement cognitif est dépendant de la langue. À partir de leur analyse, Wassmann et Dasen (1998) optent pour un " relativisme linguistique modéré », réaffirmé récemment par Dasen et al. (2000). En effet, en balinais, le système linguistique des descripteurs géocentrés semble tellement prégnant par rapport aux descripteurs égocentrés, qu'il détermine la façon de s'exprimer (la parole) mais aussi la façon de se représenter mentalement l'espace (le cognitif). Il faut souligner qu'il semble qu'il y ait souvent, dans ces travaux, une assimilation implicite de la notion d'action, c'est-à-dire de comportements sensori-moteurs observables, avec la notion de cognition, quand elle est définie en terme de représentations mentales, quant à elles inobservables (sauf, peut-être, par les techniques d'imagerie cérébrale) et estimées être à l'origine de l'action.

Les résultats obtenus à Bali et en NouvelleCalédonie entrent toutefois en contradiction avec les théories habituelles relatives au développement des concepts d'espace chez l'enfant, telle que celle de Piaget (Piaget et Inhelder, 1948). Celle-ci décrit le passage d'une représentation spatiale égocentrée (niveau 1), «espace pratique » au niveau sensori-moteur (jusque vers 2 ans), puis exocentrée (niveau 2), " espace représenté » au niveau de la pensée concrète (jusqu'à 10-11 ans), vers un système décentré, fondé sur des axes abstraits (niveau 3), au niveau de la pensée formelle (à partir de 11-12 ans) (Bideaud et al., 1993). Il serait alors possible d'envisager à Bali une sorte d' " inversion » des stades classiques du développement (Wassmann et Dasen, 1998).

L'hypothèse que l'on se propose de mettre à l'épreuve est qu'il ne s'agit pas d'une "inversion » de stades du développement, mais que l'héritage historique et culturel des sociétés, par exemple océaniennes (versus française), produit de nos jours des modalités de représentation de l'espace « inédites » (d'un point de vue occidental) et dont les étapes de développement restent à décrire (voir aussi, Troadec, 2002 ; Troadec et al., 2002). Pour y parvenir, on se propose de réaliser trois expériences. Il s'agit d'analyser les données issues d'échantillons occidentaux (région de Toulouse, France) et de les confronter aux données issues d'échantillons polynésiens (îles de Tahiti et Moorea, Polynésie française, Océanie). L'intérêt de la comparaison des populations française et polynésienne tient au fait que la société polynésienne actuelle apparaît fortement acculturée et d'un niveau socio-économique quasi équivalent à celui de la France (à la différence de la culture balinaise, par exemple). Les systèmes scolaires y sont, au moins structurellement, les mêmes. Il ne s'agit donc plus de rendre compte de l'opposition entre des sociétés modernes versus traditionnelles, mais d'envisager le développement et l'expression actuelle, ici et là-bas, de compétences spatiales et cognitives (Troadec, 1999).

À la suite de la recherche princeps de Wassmann et Dasen (1998), réalisée auprès d'enfants et non pas seulement d'adultes, les deux dispositifs expérimentaux, inspirés des travaux du Cognitive Anthropology Research Group du Max Planck Institute for Psycholinguistics de Nimègue (Pays-Bas), doivent permettre d'examiner le type de référence spatiale utilisé préférentiellement par les enfants polynésiens et français, ainsi que leur développement. Les situations expérimentales impliquent ainsi la perception visuelle d'un stimulus spatial, le traitement d'un lexique particulier induit dans la consigne, leur mémorisation à court terme, et leur rappel afin de réaliser une action d'orientation dans l'espace et de la justifier, en réponse à une consigne verbale donnée par un adulte en contexte scolaire.

Le résultat principal attendu résulte de l'effet de l'histoire et de la culture sur les représentations actuelles de l'espace (voir Ozanne-Rivierre, 1997, pour une analyse linguistique des systèmes d'orientation spatiale dans les langues austronésiennes, dont les langues polynésiennes). Tout comme à Bali, on s'attend à ce que les enfants polynésiens activent préférentiellement un système spatial géocentré ou absolu (niveau 3), dès 5 ans, et à ce que les enfants français activent inversement un système spatial égocentré ou relatif (niveau 1-2). La confrontation des résultats avec les données existantes (balinaises, indiennes, népalaises, kanak, mexicaines, etc.) devrait permettre - par la suite - une généralisation transculturelle. Le deuxième type de résul- 
tats résulte de l'effet de l'âge. Il s'agit, notamment par l'analyse qualitative des justifications verbales des actions d'orientation des enfants, de déterminer, dans la mesure du possible, les étapes d'un développement des systèmes de représentation égocentré et géocentré. Un troisième type de résultats résulte de l'effet d'une induction verbale par l'adulte d'un vocabulaire relatif à un système donné de représentation spatiale (égocentré versus géocentré). La question sousjacente est celle des rapports entre le langage et son influence sur le comportement. Il s'agit aussi d'apprécier la « présence » des deux systèmes de représentation dans la « boîte à outils » mentale de tous les enfants (niveau 1-2 et niveau 3 ) et aussi leur mise en œuvre, ou actualisation/ inhibition, en un contexte de communication particulier.

\section{EXPÉRIENCE No 1 - CONTEXTE URBAIN POLYNÉSIEN}

\section{Population}

L'échantillon est composé de 89 enfants issus d'une école maternelle et d'une école primaire de deux quartiers de la ville de Papeete (Mama'o et Pina'i). Ces enfants peuvent être considérés comme étant de culture polynésienne, telle qu'elle peut être définie de nos jours (Troadec, 1994). Globalement, le niveau socio-économique des familles est moyen, voire peu élevé. $\mathrm{La}$ langue utilisée pour l'expérience est le français. Les enfants ont été tirés au sort parmi ceux qui fréquentent les deux écoles, à l'exclusion de quelques enfants présentant de très grosses difficultés scolaires.

Trois groupes d'enfants sont constitués : 29 élèves de la Section des Grands (SG), 30 élèves de Cours Élémentaire $1^{\text {ère }}$ année (CEI) et 30 élèves de Cours Moyen $2^{\text {ème }}$ année (CM2). Les enfants de SG ont entre 4 ans 11 mois et 5 ans 10 mois, au moment de la passation. L'âge moyen du groupe est de 5 ans 5 mois. Les enfants de CE1 ont entre 6 ans 11 mois et 8 ans 10 mois, au moment de la passation. L'âge moyen du groupe est de 7 ans 5 mois. Les élèves de CM 2 ont entre 9 ans 11 mois et 12 ans 8 mois. L'âge moyen du groupe est de 10 ans 10 mois.

Chaque groupe d'enfants est divisé en deux sous-groupes, selon la modalité de l'induction verbale dans la consigne (relative ou bien absolue).

\section{Matériel}

L'organisation spatiale de l'épreuve consiste en deux salles contiguës de l'école. Les deux salles communiquent par une porte. Les enfants ne peuvent pas voir l'intérieur d'une salle lorsqu'il sont dans l'autre. Les rideaux de chaque salle sont tirés, de telle manière que les enfants ne voient pas non plus l'extérieur du bâtiment. Dans chaque salle, on dispose une table orientée selon l'axe géocentré choisi pour l'expérience :

$$
\text { Côté « montagne » } \leftarrow \text { Table } \rightarrow \text { Côté « mer » }
$$

Dans la première salle, les enfants sont assis à la première table de telle manière que le côté « mer» de l'île de Tahiti soit à leur droite et le côté "montagne» à leur gauche. Les enfants sont ensuite assis à la deuxième table, dans la deuxième salle, après une rotation de $180^{\circ}$, c'està-dire que le côté " mer » est maintenant à leur gauche, et inversement pour le côté "montagne ».

Le matériel est composé de cinq animaux : un chien, un chat, un cochon, une vache et un cheval. Il s'agit de jouets réalistes en plastique, que l'on trouve facilement dans le commerce. Tous ces animaux peuvent avoir fait l'objet d'expériences diverses par les enfants de Tahiti. On choisit trois animaux parmi les cinq, pour composer chaque item de l'épreuve :

Entraînement : chat, chien, vache ;

- Item 1: chat, cochon, cheval ;

- Item 2: cochon, chien, cheval;

- Item 3 : chat, cochon, vache;

- Item 4 : chat, chien, cheval;

- Item 5 : cochon, chien,vache.

\section{Procédure}

\section{Entraînement}

Sur la première table, on demande à l'enfant de dénommer tous les animaux. On met ensuite les trois animaux choisis pour l'item à la queue leu leu (Animals in a row) et " regardant» côté montagne. On dit à l'enfant de se rappeler comment les animaux sont placés et où ils regardent. On ramasse les animaux et on les donne à l'enfant. On lui demande alors de les placer comme ils étaient avant. Si l'enfant ne reproduit pas l'alignement, on répète l'item-test en ajoutant que les animaux regardent tous du même côté, mais sans utiliser de termes spécifiques relatifs à l'espace. Puis, on passe à l'item 1 . 


\section{Épreuve}

Pour chaque item, on place les trois animaux choisis sur la table 1 , dans la première salle. On induit verbalement un mode de représentation de la situation, en disant :

- Condition 1 - Induction verbale absolue : « Tu vois, tous les animaux regardent côté mer (versus côté montagne) »;

- Condition 2-Induction verbale relative : « Tu vois, tous les animaux regardent à gauche (versus à droite)».

On demande à l'enfant de bien se les rappeler, puis on couvre les animaux avec une feuille de papier. On déplace l'enfant à la table 2, dans la deuxième salle. On lui donne trois animaux identiques aux précédents et on lui demande de les placer comme ceux qui sont sur l'autre table. Pour les deux items 4 et 5, on demande à l'enfant de dire comment il fait pour se rappeler comment sont placés les animaux.

\section{Résultats}

\section{Cotation des réponses}

Conformément à la proposition de Wassmann et Dasen (1998), les réponses des enfants sont cotées soit A (pour Absolue) dans le cas d'une orientation géocentrée de la file d'animaux, soit $\mathrm{R}$ (pour Relative) dans le cas d'une orientation égocentrée. La cotation est appliquée ainsi lorsque les 5 réponses aux 5 items sont de même type, mais aussi lorsque 1 seul item sur 5 est de l'autre orientation. Dans le cas de protocoles où seulement 3 items sur 5 sont de la même orientation, et donc 2 de l'autre, les protocoles pourraient être cotés soit $A$ soit $R$, et traités séparément. Dans cette expérience, ils représentent près de $30 \%$ de l'ensemble (24 sur 89 ). Nous faisons ici le choix d'assimiler ces protocoles aux protocoles A et R, tout comme Dasen et al. (2000), parce qu'ils rendent compte d'une même orientation majoritaire.

\section{Effet de l'induction verbale}

Globalement, dans le cas d'une induction verbale relative, $82 \%$ des réponses sont d'orientation relative ( 37 protocoles sur 45 ) et $18 \%$ sont d'orientation absolue ( 8 protocoles sur 45). Dans le cas d'une induction verbale absolue, $57 \%$ seulement des réponses sont d'orientation absolue ( 25 protocoles sur 44 ) et $43 \%$ sont d'orientation relative (19 protocoles sur 44).
On note donc un effet de l'induction verbale sur le type d'orientation de la file d'animaux. Ceci indique un traitement effectif de l'information linguistique qui, dans une certaine mesure, « dirige » ou non l'action d'orientation dans l'espace. Cet effet apparaît très marqué pour l'induction verbale relative. On rappelle que le système d'orientation égocentré ou relatif caractérise a priori plutôt la culture française et ici scolaire. Quant à l'induction absolue, sans être nulle, elle semble avoir nettement moins d'influence sur le type d'action d'orientation spatiale. Il s'agit peut être de l'indice d'une " préférence » plus importante pour le mode d'orientation relative, dans la mesure où il est fortement « activé » malgré une information verbale absolue, dans le contexte particulier de l'expérience.

\section{Effet du sexe}

Dans le cas d'une induction verbale relative, $90 \%$ des filles font une réponse relative (18 sur 20 ) et seulement $10 \%$ d'entre elles font une réponse absolue ( 2 sur 20$)$. Quant aux garçons, $76 \%$ font aussi une réponse relative ( 19 sur 25$)$ et $24 \%$ font une réponse absolue (6 sur 25). Dans cette condition, on ne note donc pas de différence particulière de performances selon le sexe.

Dans le cas de l'induction verbale absolue, $68 \%$ des filles font malgré tout une réponse relative (13 sur 19) et seulement $32 \%$ font une réponse cohérente avec l'information verbale donnée dans la consigne. En revanche, $76 \%$ des garçons font une réponse absolue (19 sur 25), cohérente avec cette information, et $24 \%$ font une réponse relative (6 sur 25). Il y aurait donc dans cette condition un effet important du sexe sur les performances. Les filles apparaissent beaucoup moins sensibles à l'information géocentrée que les garçons. En effet, on ne note pas de différence statistiquement significative de leurs performances selon l'induction verbale, alors que c'est le cas pour les garçons.

\section{Effet du niveau scolaire}

\section{* Cas de l'induction relative}

En Section des Grands de l'école maternelle, c'est-à-dire chez les enfants de 5 ans 5 mois en moyenne, dans le cas d'une induction verbale relative, $80 \%$ des réponses sont relatives (12 sur 15). Au Cours Élémentaire 1 ère année de l'école primaire, c'est-à-dire chez les enfants de 7 ans 5 mois en moyenne, les résultats apparaissent assez similaires. $73 \%$ des réponses sont relatives (11 sur 15 ) et seulement $27 \%$ sont absolues (4 sur 15). Ensuite, au Cours Moyen $2^{\text {ème }}$ année, c'est- 
à-dire chez les enfants de 10 ans 10 mois en moyenne, $93 \%$ d'entre eux font des réponses relatives (14 sur 15). Il n'y a donc pas d'effet particulier du niveau scolaire, ou bien de l'âge, dans cette condition.

L'examen des justifications des réponses permet de préciser que, dans le cas d'une action d'orientation des animaux égocentrée, si la référence des termes de droite et de gauche n'apparaît pas bien maîtrisée en SG, elle semble le devenir plus nettement en CEI et en CM2.

Lorsque les enfants de SG font inversement des réponses géocentrées, les justifications sont $\mathrm{du}$ même type que dans le cas des protocoles précédents, c'est-à-dire que les enfants utilisent le vocabulaire égocentré induit par la consigne. Toutefois, la référence des termes de droite et de gauche n'apparaît pas bien maîtrisée et source d'incertitudes. $\mathrm{Au}$ CE1 et au CM2, les enfants utilisent aussi le vocabulaire égocentré induit verbalement. Si on peut supposer que ces termes sont mieux maîtrisés quant à leur référence, ils commettent de nombreuses erreurs du fait d'une action d'orientation absolue. Par exemple, si les animaux sont orientés à gauche sur la table 1 (et côté mer), l'enfant les oriente à droite sur la table 2 (c'est-à-dire toujours côté mer) mais il dit qu'ils regardent à gauche, car pour lui, l'orientation des animaux reste la même (gauche $=$ côté mer).

\section{* Cas de l'induction absolue}

Dans le cas d'une induction verbale absolue, on observe un effet important du niveau scolaire, ou bien de l'âge des enfants. En SG, si seulement $14 \%$ font une réponse absolue ( 2 sur 14 ), $67 \%$ font désormais de même au ce1 (10 sur 15), et enfin $87 \%$ font une réponse conforme à l'induction verbale, au CM2 (13 sur 15).

En SG, il semble que les réponses géocentrées soient justifiées par une certaine maîtrise de la signification du vocabulaire géocentré, induit par la consigne (mer/montagne). Au CEl, les enfants utilisent systématiquement le vocabulaire géocentré qui a été induit. Toutefois, la maîtrise de la référence des termes de côté mer et de côté montagne pour justifier une action d'orientation, pose encore de nombreux problèmes. Au CM2, les réponses apparaissent désormais justifiées par une utilisation adéquate des termes géocentrés. Sous cette condition, on observe donc une évolution de la maitrise d'une représentation de l'espace géocentré, qui conjoint de façon cohérence une action d'orientation avec le lexique pertinent. Cette évolution apparaît d'abord chez les garçons, dès la SG, mais surtout en CE1, où de nombreuses erreurs sont encore commises, puis chez les filles et au CM2, où très peu d'erreurs surviennent alors.

Dans le cas des réponses inversement égocentrées, on note un effet que l'on peut qualifier de pragmatique, c'est-à-dire lié à la situation d'interaction entre l'adulte et l'enfant. Dans la mesure où les termes de côté mer et de côté montagne ont été introduits dans la communication, ils deviennent pertinents pour exprimer l'action d'orientation dans l'espace réalisée par l'enfant qui, elle, est relative. Les enfants utilisent les termes côté mer / côté montagne pour désigner leur droite ou bien leur gauche. Cet effet n'exclut pas une connaissance de l'axe écologique géocentré effectif (direction de la mer et de la montagne) mais il ne permet pas l'établissement d'une différenciation des deux systèmes de représentations : l'un probablement lié à la culture scolaire, à laquelle les filles semblent plus sensibles que les garçons, l'autre à la culture polynésienne quotidienne.

Pour résumer, s'il n'y a pas de différence statistiquement significative des performances des enfants polynésiens de SG de la ville de Papeete, quelle que soit l'induction verbale, c'est-à-dire que leurs actions d'orientation dans l'espace sont essentiellement égocentrées, on note une différence des performances au CE1 qui s'accroît considérablement au CM2. À ces deux niveaux scolaires, l'information verbale induite dans la consigne apparaît mieux « diriger » et cela progressivement, l'action d'orientation d'objets dans l'espace.

\section{EXPÉRIENCE No 2 - CONTEXTE RURAL POLYNÉSIEN}

\section{Population}

L'échantillon est composé de 96 enfants d'une école maternelle et d'une école primaire d'un district de l'île de Moorea (Haapiti). Comme pour l'échantillon précédent, les enfants peuvent être considérés comme étant de culture polynésienne telle qu'elle peut être définie de nos jours (Troadec, 1994). La langue utilisée pour l'expérience est aussi le français. Les enfants ont été tirés au sort parmi ceux qui fréquentent les deux écoles, à l'exclusion de quelques enfants présentant de très grosses difficultés scolaires.

Trois groupes d'enfants sont constitués : 40 élèves de la Section des Grands (SG), 32 élèves de Cours Élémentaire $1^{\text {ère }}$ année (CE1) et 24 élèves de Cours Moyen $2^{\text {ème }}$ année (CM2). Les enfants 
de SG ont entre 5 ans 0 mois et 5 ans 11 mois, au moment de la passation. L'âge moyen du groupe est de 5 ans 6 mois. Les enfants de CE1 ont entre 7 ans 0 mois et 8 ans 9 mois. L'âge moyen du groupe est de 7 ans 9 mois. Par erreur, les dates de naissance des enfants de CM2 n'ont pas été relevées, ce sont donc des enfants de 10-11 ans environ.

Chaque groupe d'enfants est divisé en quatre sous-groupes, selon le type d'objets utilisé dans l'épreuve (les voitures ou bien les cartes) et la modalité de l'induction verbale dans la consigne (relative ou bien absolue).

\section{Matériel}

L'organisation spatiale de l'épreuve consiste en deux tables, séparées par un tableau mobile et disposées dans une salle de l'école. Les rideaux de la salle sont tirés, de telle manière que les enfants ne voient pas l'extérieur du bâtiment. Ils ne peuvent pas non plus voir ce qui se trouve sur l'une des tables lorsqu'ils sont assis à l'autre. Comme dans l'expérience 1, on oriente les deux tables selon l'axe géocentré choisi pour l'expérience :

$$
\text { Côté « montagne » } \leftarrow \text { Table } \rightarrow \text { Côté « mer » }
$$

Le matériel est composé d'une part de petites voitures, d'autre part de cartes plastifiées (Chips). Les voitures sont des jouets réalistes, en métal, que l'on trouve dans le commerce. L'orientation d'une voiture dans l'espace apparaît liée à la direction qu'elle prend en avançant, par rapport à un observateur, et cette connaissance spatiale fait partie des expériences quotidiennes vécues par les enfants. Les cartes sont des supports en carton sur lesquels se trouvent des formes géométriques de couleurs différentes : un petit rond rouge et un grand carré bleu. L'orientation des deux objets, l'un par rapport à l'autre, et sur la carte, n'est pas donnée a priori. Elle résulte d'une norme culturelle qui consiste à associer une certaine orientation des deux objets sur la carte, dite exocentrée, par rapport à la position d'un locuteur, selon des énoncés du type :

- le rond est « sur » le carré ;

— le rond est « sous » le carré ;

— le rond est « à côté » du carré.

Cette norme rend l'orientation "évidente» dans le cas d'une interaction sociale entre un adulte et un enfant de niveau scolaire préélémentaire et élémentaire mais il est important de noter que, même largement partagée, cette évidence résulte d'un apprentissage culturel particulier, notamment scolaire. Ainsi, il peut être intéressant de distinguer des objets pour lesquels l'orientation dans l'espace est plus (les cartes) ou moins (les voitures) culturellement normée.

Le matériel est composé de cinq petites voitures en métal, toutes différentes les unes des autres (quatre « $4 \mathrm{x} 4$ » et une voiture de sport) et de cinq cartes identiques $(10,5 \mathrm{~cm} \times 10,5 \mathrm{~cm})$. Sur chaque carte, il y a un dessin géométrique représentant le même grand carré bleu $(5,5 \mathrm{~cm} \times 5,5 \mathrm{~cm})$ surmonté d'un petit cercle rouge $(\mathrm{D}=3 \mathrm{~cm})$.

\section{Procédure}

\section{Entraînement}

Sur la première table, on place les cinq cartes (versus les cinq voitures) dans la même direction par rapport aux enfants : les carrés "surmontés» du cercle et les voitures allant «droit devant $»$. On demande aux enfants de décrire la situation. On prend l'une des cartes (versus la voiture de sport) et on la place dans une direction différente. Le cercle est sur le côté gauche du carré et la voiture va "de côté » (rotation de $90^{\circ}$ ). L'expérimentateur dit ensuite : « Maintenant, une des cartes (versus une des voitures) est différente des autres. Peux-tu voir pourquoi ? Tu dois te rappeler comment est cette carte (versus cette voiture) ». On cache la carte ou la voiture avec un morceau de tissu et on place les quatre autres devant les enfants de telle sorte qu'elle forment une croix et que chaque cercle et chaque voiture soit dans une orientation différente. "Maintenant, regarde ces cartes (versus ces voitures) et dis moi quelle est celle qui est comme celle que tu as vu avant ». On arrête lorsque les enfants ont compris.

\section{Épreuve}

On place une carte (versus la voiture de sport) sur la première table et on demande aux enfants de bien s'en rappeler. On induit verbalement une orientation relative ou bien absolue.

- Condition 1 - Induction relative : " Tu vois le rond est à droite (versus la voiture va à droite) » ou « Tu vois le rond est à gauche (versus la voiture va à gauche) »;

- Condition 2 - Induction absolue : « Tu vois le rond est côté mer (versus la voiture va côté mer) » ou « Tu vois le rond est côté montagne (versus la voiture va côté montagne) ».

On cache la carte ou la voiture avec un morceau de tissu et on invite les enfants à aller à la deuxième table. Sur celle-ci, on dispose les quatre 
autres cartes ou les quatre autres voitures en croix, chacune dans une orientation différente. Les enfants doivent choisir la carte ou la voiture qui correspond, selon eux, à celle qui est sur la première table. On réalise la procédure avec cinq items, ou dispositions différentes. On demande aux enfants de justifier leurs réponses pour les items 3,4 et 5 .

\section{Résultats}

\section{Cotation des réponses}

Comme dans l'expérience 1, les réponses des enfants sont cotées soit A (pour Absolue) dans le cas d'une orientation géocentrée, soit $\mathrm{R}$ (pour Relative) dans le cas d'une orientation égocentrée. Onze protocoles n'ont toutefois pas pu être cotés en A ou bien R. Ces autres protocoles semblent indiquer une difficulté importante de l'enfant à comprendre et répondre à la question posée, avec un choix quelque peu aléatoire de chaque orientation possible l'une après l'autre. On trouve ces protocoles majoritairement en SG (8 sur 11).

\section{Effet de l'induction verbale}

Globalement, dans le cas d'une induction verbale relative, $19 \%$ des réponses sont d'orientation relative ( 8 protocoles sur 41 ) et $81 \%$ sont d'orientation absolue (33 protocoles sur 41). Dans le cas d'une induction verbale absolue, $89 \%$ des réponses sont d'orientation absolue (39 protocoles sur 44) et seulement $11 \%$ sont d'orientation relative (5 protocoles sur 44 ). Les autres protocoles se répartissent en $64 \%$ (7 sur 11) pour l'induction relative et $36 \%$ (4 sur 11) pour l'induction absolue.

On ne note donc pas d'effet de l'induction verbale sur le type d'orientation choisie par les enfants. Quelle que soit l'information qui leur est donnée dans la consigne, une large majorité d'entre eux (plus de $80 \%$ ) fait une réponse selon l'axe géocentré. Ceci peut être l'indice d'une " préférence » culturelle plus importante pour ce mode d'orientation, quelle que soit l'information verbale reçue, et dans ce contexte particulier. Comme on peut le constater, ces résultats sont très différents de ceux obtenus à Papeete.

\section{Effet du sexe}

Dans le cas d'une induction verbale relative, $71 \%$ des filles font une réponse absolue (12 sur 17) et $29 \%$ d'entre elles font une réponse relative (5 sur 17). Quant aux garçons, $88 \%$ font aussi une réponse absolue ( 21 sur 24$)$ et seulement $12 \%$ font une réponse relative ( 3 sur 24 ). Les filles apparaissent donc un petit peu plus sensibles à l'information verbale relative que les garçons, sans pour autant qu'il y ait de différences statistiquement significatives.

Dans le cas de l'induction verbale absolue, on ne note pas non plus de différence particulière selon le sexe. $14 \%$ des filles ( 3 sur 21 ) et $9 \%$ des garçons (2 sur 23) font une réponse relative, c'est-à-dire non conforme à l'information verbale qui leur est donnée. Ainsi, $86 \%$ des filles (18 sur 21 ) et $91 \%$ des garçons ( 21 sur 23 ) font une réponse cohérente avec l'induction absolue. Les autres protocoles se répartissent en $73 \%$ ( 8 sur 11) pour les filles et $27 \%$ ( 3 sur 11) pour les garçons.

\section{Effet du type d'objets}

Dans le cas d'une induction verbale absolue, on n'observe pas de différence de performances selon le type d'objets utilisés. Avec les cartes, $91 \%$ des réponses sont absolues (20 sur 22), et $86 \%$ le sont avec les voitures (19 sur 22). En revanche, on relève une légère différence de traitement des deux types d'objets dans le cas d'une induction verbale relative, bien que celle-ci ne soit pas statistiquement significative. Si seulement $15 \%$ des réponses sont relatives avec les voitures ( 3 sur 20$), 24 \%$ le sont lorsqu'il s'agit des cartes (5 sur 21). Ceci peut être l'expression faible d'un statut des cartes un peu plus sensible au contexte scolaire, dans la mesure où il tend à renforcer une représentation égocentrée de l'espace, issue des normes culturelles occidentales, par rapport aux voitures qui font l'objet d'expériences de la vie quotidienne, normées par la culture polynésienne non scolaire. Les autres protocoles se répartissent de façon égale en $45 \%$ ( 5 sur 11 ) pour les cartes et $55 \%$ (6 sur 11) pour les voitures.

\section{Effet du niveau scolaire}

* Cas de l'induction relative

En Section des Grands de l'école maternelle, c'est-à-dire chez les enfants de 5 ans 6 mois en moyenne, dans le cas d'une induction verbale relative, $94 \%$ des réponses sont absolues (14 sur 15). Au Cours Élémentaire 1 ère année de l'école primaire, c'est-à-dire chez les enfants de 7 ans 9 mois en moyenne, et toujours dans le cas d'une induction verbale relative, les résultats apparaissent assez différents. $36 \%$ des réponses sont relatives ( 5 sur 14 ) et $64 \%$ sont absolues ( 9 sur 14 ). Cette différence se réduit ensuite au Cours 
Moyen $2^{\text {ème }}$ année, c'est-à-dire chez les enfants de $10-11$ ans, où $83 \%$ d'entre eux font des réponses absolues (10 sur 12) et $17 \%$ des réponses relatives ( 2 sur 12$)$. Il y a donc un léger effet du niveau scolaire dans cette condition (au CE1). Cette différence n'est toutefois pas statistiquement significative.

Dans le cas des actions d'orientation relative, au CE1 et au CM2, les justifications des réponses reprennent généralement le vocabulaire utilisé et indiquent une maîtrise de l'orientation égocentrée (droite/gauche). Globalement, l'information verbale induite par la consigne apparaît donc traitée par les enfants. Les quatre directions sont alors dénommées correctement en termes de gauche et de droite, et de devant et de derrière (beaucoup moins fréquemment, en termes de haut et de bas). Pour ces quelques cas, plus fréquents chez les filles et au CE1, on observe une bonne adéquation entre l'action égocentrée d'orientation d'objets dans l'espace et l'utilisation d'un vocabulaire pertinent pour rendre compte des quatre directions.

Pour ce qui concerne les actions d'orientation absolue, en SG, les justifications des réponses reprennent aussi le vocabulaire relatif utilisé dans l'induction verbale. Toutefois, les termes de gauche et de droite, lorsqu'ils sont utilisés, correspondent alors à un axe géocentré (mer/ montagne), dans la mesure où leur référence apparaît systématiquement inversée selon l'orientation égocentrée. Au CE1 et au CM2, les justifications sont du même type que précédemment. Les quatre orientations sont alors désignées par les termes relatifs de droite et de gauche, induits par la consigne, et très souvent inversés selon l'axe égocentré (11 fois sur 19). Contrairement aux protocoles relatifs précédents, on relève de plus une variabilité interindividuelle importante des désignations de l'axe «vertical». Pour ces enfants, l'induction par l'adulte de certains termes dans la consigne « fixe » le vocabulaire à utiliser dans le cadre de la communication. La référence de ces termes, qui n'est pas induite quant à elle par l'adulte, est alors celle qui est activée préférentiellement par l'enfant. Dans ce contexte particulier, les expressions linguistiques de gauche et de droite peuvent servir, toutefois de façon non systématique, à désigner une action d'orientation absolue (mer/montagne).

\section{* Cas de l'induction absolue}

Dans le cas d'une induction verbale absolue, il n'apparaît pas de différence liée au niveau scolaire ou bien à l'âge des enfants. En SG, 88 \% font une réponse absolue (15 sur 17), au CE1, $87 \%$ font de même (13 sur 15), et au CM2, $92 \%$ font une réponse conforme à l'induction verbale (11 sur 12).

Lorsque l'orientation des objets est absolue, en SG, au CE1 et au CM2, les justifications des réponses utilisent correctement les expressions absolues, induites par la consigne. La désignation des quatre orientations, relevée seulement au CE1 et au CM2, se fait par l'utilisation correcte des expressions côté mer et côté montagne, pour le premier axe et par une variabilité interindividuelle importante de termes relatifs au deuxième. On peut penser que, dans le cadre de l'interaction sociale entre l'adulte et l'enfant que constitue la situation expérimentale, l'action d'orientation géocentrée, activée par une majorité d'enfants polynésiens, peut être exprimée de façon partagée grâce aux termes induits par l'adulte dans le cadre de la communication. Dans la mesure où aucun terme particulier n'est introduit pour désigner l'axe "vertical», on observe une grande variabilité de productions linguistiques pour en rendre compte.

Lorsque l'orientation des objets est relative, en $\mathrm{SG}$, les justifications des réponses sont en terme de « je ne sais pas ». Au CE1 et au CM2, celles-ci utilisent les expressions absolues induites par la consigne, mais pour justifier une orientation relative. On peut penser que l'action égocentrée d'orientation dans l'espace, apparemment activée par ces quelques enfants, n'est pas facilement exprimable par un langage commun du type "gauche/droite et devant/derrière", compte tenu de l'induction préalable par l'adulte d'un vocabulaire géocentré (mer/montagne).

Pour résumer, quel que soit le niveau scolaire et le type d'induction verbale, il n'y a pas de différences statistiquement significatives des performances chez les enfants de Moorea. Leurs actions d'orientation d'objets dans l'espace sont essentiellement géocentrées. On précise enfin que l'on a fait aussi passer cette épreuve à des enfants de culture française (Beaumont-de-Lomagne) en France. Globalement, contrairement aux résultats obtenus à Moorea, en CP, on observe une différence statistiquement significative des performances selon l'induction verbale, c'est-à-dire que les performances apparaissent « orientées » et cohérente avec le type de lexique relatif à l'espace et utilisé par l'adulte. Ensuite, il n'y a plus de différence au CE2, où une action d'orientation spatiale égocentrée est réalisée majoritairement, quelle que soit l'information verbale induite par l'adulte (l'information linguistique géocentrée a alors beaucoup moins d'effet sur l'action d'orientation d'objets). 


\section{DISCUSSION}

En milieu urbain polynésien (ville de Papeete) et dans l'épreuve proposée (Animaux), les enfants de 5-6 ans orientent massivement les objets selon un axe égocentré (droite/gauche). On peut estimer qu'il s'agit-là d'une influence de la scolarité et conjointement du milieu urbain. À 7-9 ans toutefois, l'induction verbale apparaît mieux traitée d'un point de vue sémantique, dans le sens où les actions d'orientation spatiale lui sont désormais cohérentes. Ce phénomène qui intéresse d'abord les seuls garçons, s'accroît avec l'âge. À 10-12 ans, cette adéquation langage/ action est quasi parfaite chez tous les enfants. On peut donc en conclure à une maîtrise, à cet âge, des deux systèmes de représentation spatiale.

En milieu rural polynésien (île de Moorea) et dans l'épreuve proposée (Cartes et Voitures), les résultats apparaissent très différents des précédents. En effet, dès 5-6 ans, l'orientation spatiale des objets est réalisée majoritairement selon un axe géocentré (mer/montagne), quelle que soit l'induction verbale. Des résultats similaires ont été obtenus récemment par Cablitz (2002) auprès d'enfants des îles Marquises. Les actions d'orientation géocentrée qui suivent une information verbale égocentrée sont plus fréquentes chez les garçons que chez les filles. Si on note une tendance à mieux intégrer l'information égocentrée à 7-9 ans, mais seulement chez les filles, le phénomène apparaît assez stable jusqu'à 10-12 ans. Dans ce contexte, l'information verbale égocentrée (en termes de droite et de gauche) n'apparaît donc pas sémantiquement pertinente. Malgré l'acculturation scolaire, les enfants de l'île de Moorea semblent développer une représentation préférentiellement géocentrée de l'espace. Celle-ci apparaît adaptée au contexte écologique insulaire et au milieu socio-économique rural polynésien.

Enfin, les enfants ruraux français de 6-7 ans, dans l'épreuve proposée (Cartes) semblent traiter adéquatement l'information verbale qui est induite dans la consigne. Toutefois, à 9-10 ans, une tendance à orienter les objets selon un axe égocentré, malgré une information inversement géocentrée, émerge, et principalement chez les filles. On peut voir là l'effet de la culture française où prédomine une représentation égocentrée de l'espace, associée à la scolarisation. De ce point de vue, les enfants les plus jeunes apparaissent nettement plus pluralistes que leurs aînés. Selon un autre "chemin », mais de façon similaire à celui de l'île de Moorea, la variabilité des représentations spatiales semble, dans ce contexte, se réduire progressivement avec l'âge à une représentation préférentielle, contrairement au contexte urbain polynésien (Papeete) où la pluralité apparaît mieux maîtrisée.

Dans leur recherche, réalisée en Inde et au Népal, Dasen et al. (2000) ont fait passer (entre autres) les deux épreuves des "Animaux à la queue leu leu » (Animals in a row) et des «Cartes »(Chips) aux mêmes enfants. Les résultats obtenus par ces auteurs montrent une variabilité des performances selon le type de tâche. Il en ressort que «l'encodage est, en fait, spécifique des situations proposées » (p. 21). L'épreuve des Animaux induirait un encodage plutôt absolu. On peut effectivement penser que la représentation de la file des trois animaux est construite plus aisément selon une orientation par rapport à l'espace écologique dans lequel les animaux sont sensés se déplacer. L'épreuve des Cartes, quant à elle, induirait un encodage plutôt relatif, du fait d'une orientation plus arbitraire des deux figures géométriques l'une par rapport à l'autre. Cela dit, le fait que le petit cercle soit « sur» le grand carré peut apparaître plus écologique que l'inverse. Quoiqu'il en soit, on ne retrouve pas le même type d'effet auprès des échantillons polynésiens. En milieu urbain (Papeete), l'épreuve des Animaux induit un encodage relatif massif, lors d'une induction verbale elle aussi relative, et en milieu rural (île de Moorea), l'épreuve des Cartes induit un encodage absolu massif, quelle que soit l'induction verbale. Dans cette épreuve particulière, on ne trouve d'ailleurs pas d'effet statistiquement significatif selon le matériel utilisé (cartes ou voitures). S'il y a une très légère tendance à un traitement relatif des Cartes, l'utilisation des Voitures, qui peuvent s'apparenter aux Animaux, induit le même type de performances que les Cartes. Conscients de cette variabilité des performances selon les tâches et aussi selon les contextes écoculturels, on tente malgré tout une analyse comparative prudente, en terme de " cheminements » différents du développement cognitif en contextes écoculturels.

En revanche, si Dasen et al. (2000) n'ont pas induit dans la consigne de descripteurs linguistiques spécifiques (égocentrés versus géocentrés), les résultats obtenus en Inde et au Népal correspondent pour une large part à ceux obtenus en Polynésie. À l'épreuve des Animaux, en milieu indien urbain, on observe une augmentation progressive des performances géocentrées avec l'âge (de $55 \%$ des protocoles à $4-5$ ans, à $75 \%$ à 12-14 ans). À la même épreuve, dans les milieux ruraux et dans tous les cas (indien et népalais), 
les protocoles absolus sont les plus fréquents. En milieu rural indien, à l'épreuve des Cartes, on observe le même type d'effet ( $50 \%$ de protocoles absolus à $4-5$ ans et $76 \%$ à 12-14 ans). Il en ressort, selon les auteurs, "que le système d'orientation spatiale, et le langage qui y réfère, apparaît adapté aux différents contextes écologiques » (p. 29). Les contextes ruraux népalais et indien induisent une utilisation préférentielle d'une représentation géocentrée, comme à Moorea, alors que le contexte urbain indien suscite une utilisation plus variée des systèmes de représentations, et est le seul où l'utilisation de références spatiales relatives (droite et gauche) est observée, comme à Papeete.

Pour le cas des recherches présentées ici, l'effet de l'induction d'un vocabulaire relatif à un espace égocentré ou bien géocentré apparaît assez différent selon les contextes écoculturels envisagés. Cet effet permet de rendre compte des relations complexes entre le langage et l'action sensorimotrice, ainsi que leur développement. En l'occurrence, il apparaît que les actions d'orientation d'objets dans l'espace ne correspondent pas systématiquement à ce que les descripteurs linguistiques permettent d'anticiper. Cette correspondance action/langage semble s'apprendre progressivement, et la configuration qu'elle prend, à partir d'un certain âge, dépend de nombreux facteurs liés aux situations, à l'écologie et à la culture. Ceci permet, selon nous, de relativiser fortement l'hypothèse d'un relativisme linguistique, au moins chez l'enfant en développement.

\section{BIBLIOGRAPHIE}

Ascher, M., 1998. Mathématiques d'ailleurs. Nombres, formes et jeux dans les sociétés traditionnelles (trad. de l'anglais, 1991), Paris, Seuil.

BAudelot, C., 1991 [2 $2^{\text {ème }}$ édition, 1992]. Sapir Edward, in P. Bonte et M. Izard (eds), Dictionnaire de l'ethnologie et de l'anthropologie, Paris, PUF.

Bideaud, J., O. Houdé et J.-L. Pedinielli, 1993. L'homme en développement, Paris, PUF.

BOWERMAN, M. and S. LEVINSON (eds), 2000. Language Acquisition and Conceptual Development, Cambridge, Cambridge University Press.

Brown, P. and S. Levinson, 2000. Frames of Spatial Reference and Their Acquisition in Tenejapan Tzeltal in L. Nucci and G. Saxe (eds), Culture, Thought, and Development, Mahwah, Lawrence Erlbaum, pp. 167-197.

Cablitz, G., 2002. The Role of Ego and Perceptual Clues in the Acquisition of a Local Landmark-
Based Absolute System in Marquesan (Oceania, French Polynesia), Communication orale, $16^{\text {ème }}$ Congrès de l'International Association for Cross-Cultural Psychology (IACCP), Yogyakarta (Indonésie).

Cottereau-Reiss, P., 1999. L'espace kanak ou comment ne pas perdre son latin! Annales de la Fondation Fyssen 14, pp. 34-45.

_, 2002. Langages d'espace : l'exemple kanak in C. Sabatier et P. Dasen (eds), Cultures, développement et éducation. Autres enfants, autres écoles, Paris, L'Harmattan, pp. 159-168.

DANZIGER, E., 1997. La variation inter-langues dans l'encodage sémantique et cognitif des relations spatiales : quelques réflexions sur les données du maya mopan, in C. Fuchs et S. Robert (eds), Diversité des langues et représentations cognitives, Paris, Éditions Ophrys, pp. 58-80.

-, 2001. Cross-Cultural Studies in Language and Thought : Is There a Metalanguage ?, in C. Moore and H. Mathews (eds), The Psychology of Cultural Experience Cambridge, Cambridge University Press, pp. 199-222.

Dasen, P., R. Mishra and S. Niraula, 2000. Ecology, Language, and Performance on Spatial Cognitive Tasks. Communication orale, $15^{\text {ème }}$ Congrès de 1'International Association for Cross-Cultural Psychology (IACCP), Pultusk (Pologne).

Levinson, S., 1996. Frames of Reference and Molyneux's Question: Cross-Linguistic Evidence, in P. Bloom, M. Peterson, L. Nadel and M. Garrett (eds), Language and Space, Cambridge, MIT Press, pp. 109-169.

Mazellier, P., vers 1965. Tahiti, Éditions Rencontre.

OZANNE-RivierRe, F., 1997. Systèmes d'orientation : quelques exemples austronésiens, in $\mathrm{C}$. Fuchs et $\mathrm{S}$. Robert (eds), Diversité des langues et représentations cognitives, Paris, Éditions Ophrys, pp. 81-92.

Peltzer, L., 1998. Représentation et structuration de l'espace en tahitien, Bulletin de la Société des Études Océaniennes 276, pp. 2-26.

Piaget, J et B. InHelder, 1948. La représentation de l'espace chez l'enfant, Paris, PUF.

Trondec, B., 1994. Demi-Tahitien : Tahitien et Demi à la fois ? Une approche psychologique de l'identité polynésienne d'aujourd'hui, Psychologie et Éducation, revue de l'Association Française de Psychologie Scolaire (AFPS), 18, pp. 43-65.

-, 1998. Psychologie du développement cognitif, Paris, Armand Colin.

_, 1999. Psychologie culturelle du développement, Paris, Armand Colin.

—, 2001a. Le modèle écoculturel : un cadre pour la psychologie culturelle comparative, Journal International de Psychologie 36, pp. 53-64.

_, 2001b. Approches culturelle et interculturelle comparative : vers une intégration de paradigmes complémentaires. Communication orale. $8^{\text {ème }}$ Congrès 
International de l'Association pour la Recherche InterCulturelle (ARIC). Genève (Suisse).

_, 2002. When Language Makes Ecological Directions Change: a Developmental Study of Spatial Orientation Among Polynesian and French Children. Communication orale. $16^{\text {ème }}$ Congrès de l'International Association for Cross-Cultural Psychology (IACCP), Yogyakarta (Indonésie).

_, à paraître. Point de vue sur l'interculturel : les schèmes d'un psychologue du développement cognitif, Bulletin de Psychologie.

Troadec, B., C. Martinot et P. Cottereau-Reiss, 2002. The Cross-Cultural Study of Diversity in
Cognitive Development: Categorization and Space, in P. Boski, F. Van de Vijver and M. Chodynicka (eds), New Directions in Cross-Cultural Psychology, Varsovie (Pologne), Polish Academy of Sciences, pp. 243-258.

Wassmann, J., 1994. The Yupno as Post-Newtonian Scientists. The Question Of What Is 'Natural' in Spatial Description, Man 29, pp. 1-24.

Wassmann, J. and P. Dasen, 1998. Balinese Spatial Orientation : Some Empirical Evidence for Moderate Linguistic Relativity, The Journal of the Royal Anthropological Institute, Man 4, pp. 689-711. 